\title{
NESSIE: an experimental search for sterile neutrinos with the CERN-SPS beam
}

\author{
Marco Roda*t \\ Padova University and Padova INFN \\ E-mail: marco.roda@pd.infn.it
}

\begin{abstract}
Recent results on neutrino oscillation with Short-Base-Line (SBL) experiments and the reanalysis of past measures, based on the recomputed antineutrino fluxes from nuclear reactors, design a picture not fully compatible with the phenomenological oscillation scenario with 3 neutrinos. A new experimental program is therefore needed to clarify the physics issue with possibly a new SBL neutrino beam at CERN. NESSIE has been proposed for the search of sterile neutrinos studying the leptons produced in CC neutrino and antineutrino interactions. The detectors consist of two magnetic spectrometers to be located in two sites: "Near" and "Far" from the proton target of the CERN-SPS beam. Each spectrometer will be complemented by an ICARUS-like LAr target in order to allow also $\mathrm{NC}$ and $v_{e} \mathrm{CC}$ interactions reconstruction.
\end{abstract}

XV Workshop on Neutrino Telescopes,

11-15 March 2013

Venice, Italy

\footnotetext{
* Speaker.

†n behalf of the NESSiE collaboration

${ }^{\ddagger}$ Neutrino Experiment with SpectrometerS in Europe
} 


\section{Physical Motivation}

Tensions in several phenomenological models grew up with experimental results on neutrino and anti-neutrino oscillations at Short-Baseline (SBL) and with the recent, carefully recomputed, anti-neutrino fluxes from nuclear reactors. Two distinct classes of anomalies have been reported.

The observation of excess electrons originated by initial $v_{\mu}$ beam from accelerators (LSND [1] and MiniBooNe [2]). At present, the LSND experiment and the MiniBooNe experiment both claim an independent $3.8 \sigma$ effect from standard neutrino physics. The LSND signal with antineutrino oscillations from an accelerator would imply an additional mass-squared difference largely in excess of the Standard Model values. The LSND signal represents a $3.8 \sigma$ effect in the $L / E$ range around $0.5 \div 1.0 \mathrm{~m} / \mathrm{MeV}$. The recent MiniBooNe result, confirming the LSND result, indicates a neutrino oscillation signal both in neutrino and anti-neutrino with $\Delta m^{2} \sim 0.01$ to $1.0 \mathrm{eV}^{2}$.

The apparent disappearance signal in the $v_{e}$ events detected from near-by nuclear reactors and from k-capture calibration sources in the solar $v_{e}$ Gallium experiments [3]. Recently a reevaluation of all the reactor anti-neutrino spectra has increased the flux by about $4 \%$ and a new value of the neutron lifetime has been reported. With such a new flux evaluations, the ratio between the observed and predicted rates decreased, leading to a deviation of $3.0 \sigma$ from unity $(99.6 \%$ confidence level). SAGE and GALLEX experiments recorded the calibration signal produced by intense artificial k-capture sources. The averaged ratio between the detected and predicted neutrino rates are consistent with each other at about $2.7 \sigma$ from unity, pointing to broad range of values centered around $\Delta m^{2} \sim 2 e V^{2}$ and $\sin ^{2}\left(2 \theta_{\text {new }}\right) \sim 0.3$.

By combining the Gallium and the reactor anomalies the non oscillation hypothesis is disfavored at $3.6 \sigma$.

\section{Detectors}

In order to observe a neutrino spectrum variation, the setup consists of two detectors which are located in two different sites: a "near" and a "far" one, respectively $472 \mathrm{~m}$ and $1623 \mathrm{~m}$ from the proton target, see Figure 2. The detectors are made by two similar spectrometers [6]. Each detector is situated downstream an ICARUS-like LAr TPC. The aim is the measurement of charge and momentum of the muons coming from the charge current neutrino interactions. The present configuration, for both the spectrometers, consists of an Air Core Magnet (ACM) followed by an Iron Core Magnet (ICM), sketched in Figure 3.

The ACM is designed to be able to measure the charge of low momentum muons, in order to distinguish neutrino and antineutrinos also at low energies. The designed magnetic field can reach $0.1 T$ using a dedicated power supply. The detector to be installed in the ACM is not defined yet, anyway one of the possible detectors consists of scintillator strips readout by SiPM, since standard phototubes are not expected to work properly because of the ACM magnetic field.

The ICM is composed of 2 arms made of 22 iron slabs. Each of the 44 iron slabs is $5 \mathrm{~cm}$ thick. The iron is magnetized with a current of $1600 \mathrm{~A}$ : the resulting magnetic field in the iron is $1.5 \mathrm{~T}$. The gaps between the slabs are instrumented with RPCs with a digital readout. Other possibilities are under investigation, such as the use of two smaller consecutive spectrometers instead of a bigger one. 


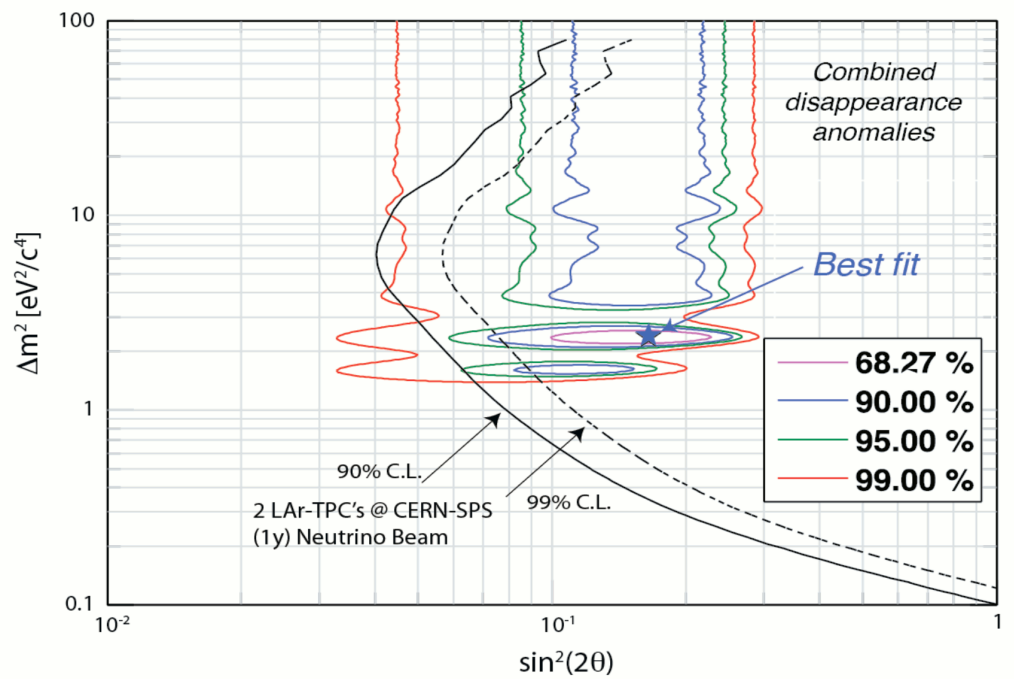

Figure 1: Colored lines: allowed regions in the $\sin ^{2}\left(2 \theta_{\text {new }}\right)-\Delta m^{2}$ plane [4] from the combination of reactor neutrino experiments, GALLEX and SAGE calibration sources experiments, MiniBooNe reanalysis of Ref. [5], and the ILL energy spectrum distortion. The data are well fitted by the $3+1$ neutrino hypothesis, while the no-oscillation hypothesis is disfavored at $99.8 \%$ C.L. The $\chi^{2}$ profiles for $\left|\Delta m^{2}\right|$ and $\sin ^{2}\left(2 \theta_{\text {new }}\right)$ (1 dof) lead to the constraints at $95 \%$ C.L. given by $\left|\Delta m^{2}\right|>1.5 \mathrm{eV}^{2}$ and $\sin ^{2}\left(2 \theta_{\text {new }}\right)=0.14 \pm 0.08$. Oscillation sensitivity in $\sin ^{2}\left(2 \theta_{\text {new }}\right) v s \Delta m^{2}$ distribution for 1 year of data taking is also showed, black lines.

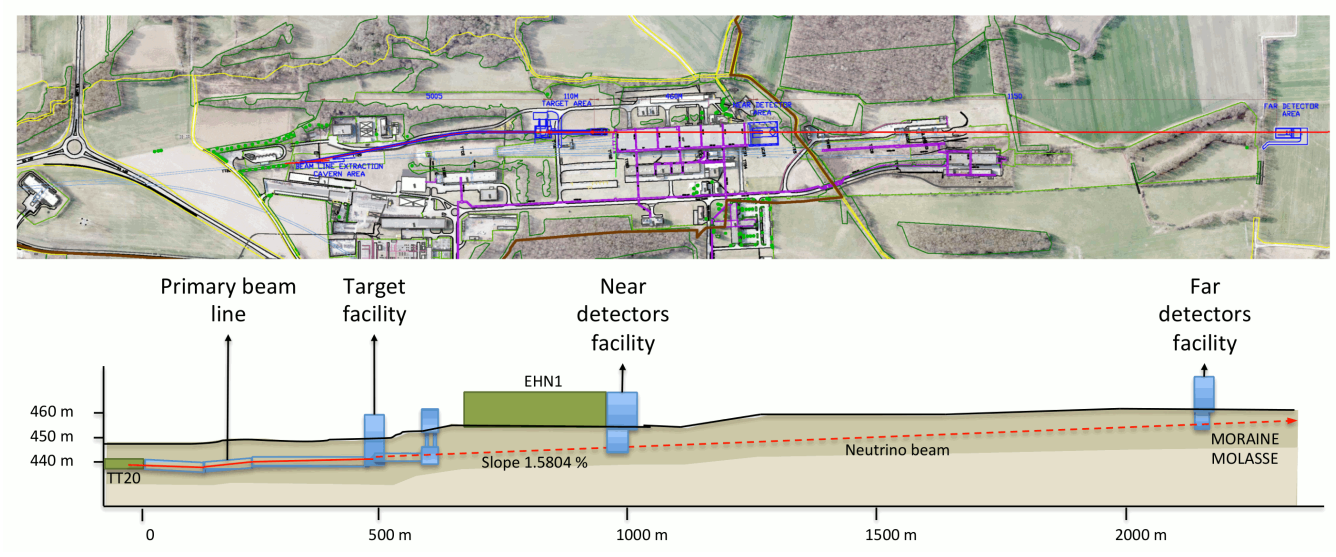

Figure 2: CERN northern area and neutrino beam facilities project. Satellite view (up) and side view (down). 


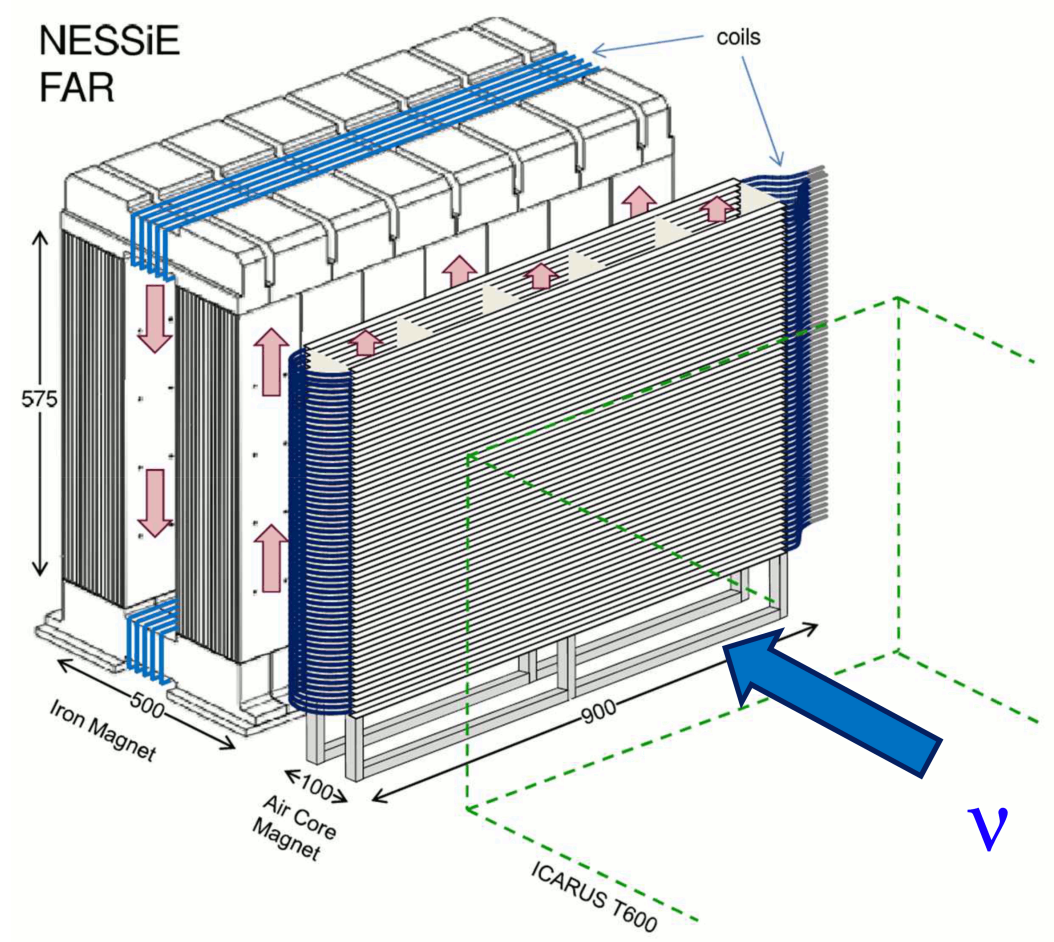

Figure 3: Detector scheme.

\section{Momentum and charge reconstruction}

The region of interest, where the oscillation is expected to be visible, ranges up to muon energies of a few $\mathrm{GeV}$.

In this kinematical region, the best momentum reconstruction is given by the energy loss of the muon in the iron slabs of the magnet. Using this method, the muon momentum can be reconstructed from few hundreds $\mathrm{MeV}$ to $3 \mathrm{GeV}$ with an uncertainty less than $6 \%$. Of course, using this method, the reconstruction is reliable only for muon stopping in the magnet. For escaping muons, the momentum can be estimated by the bending of the muon tracks in the magnetic field.

The charge reconstruction efficiency varies a lot as a function of the incoming muon energy, see the plot in Figure 5. At low energy, the best efficiency is given by the ACM: in this case the charge mis-identification is less than $0.5 \%$. From $2 \mathrm{GeV}$ on, the best charge reconstruction is given by the ICM. For both ACM and ICM the efficiency decreases: at $20 \mathrm{GeV}$ the mis-ID is around $30 \%$ for the ICM and $40 \%$ for the ACM.

\section{Data taking program and expected sensitivity}

For the $v_{\mu} \rightarrow v_{e}$ transition, with one year exposure $\left(4.5 \cdot 10^{19}\right.$ pot) at the CERN-SPS $v_{\mu}$ beam, the experiment is sensitive to $\sin ^{2}(2 \theta)$ down to $3 \cdot 10^{4}$ (for $\left|\Delta m^{2}\right|>1.5 \mathrm{eV}^{2}$ ) and $\left|\Delta \mathrm{m}^{2}\right|$ down to $0.01 \mathrm{eV}^{2}$ (for $\sin ^{2}(2 \theta)=1$ ) at $90 \%$ C.L, see plot in Figure 6. So, the parameter space region allowed by the LSND experiment is fully covered. The sensitivity has been computed assuming a 
IM arms momentum

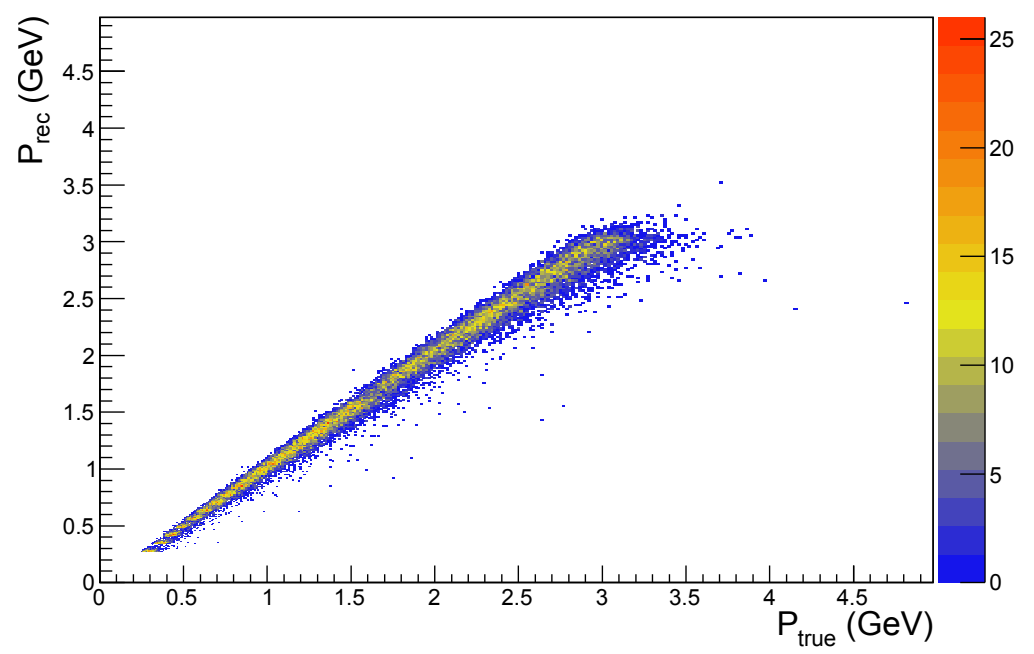

Figure 4: Momentum reconstruction for muons stopping in the detector.

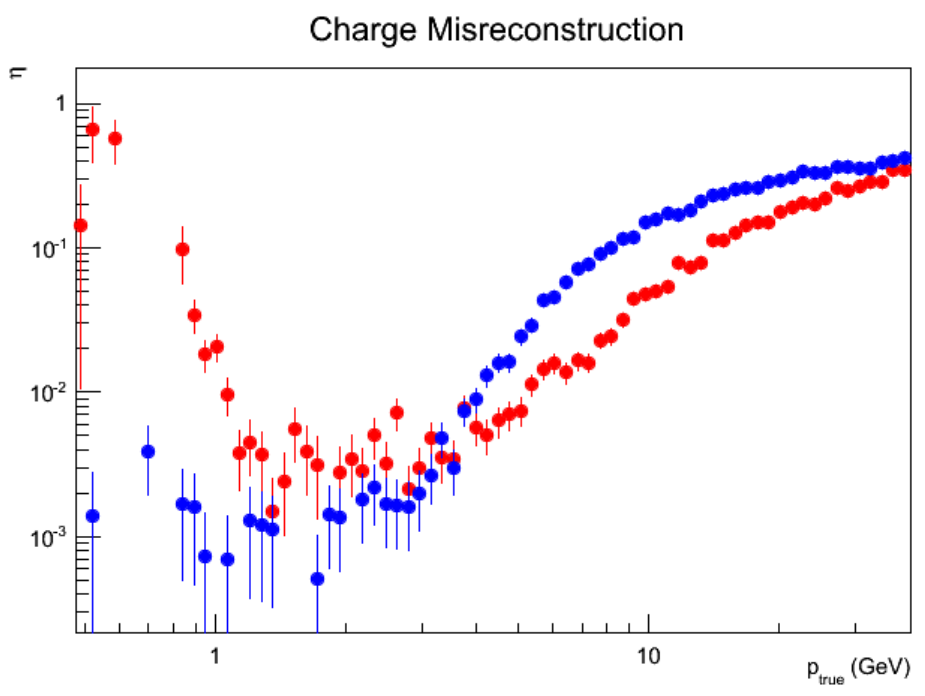

Figure 5: Charge misidentification for muons as a function of energy. The error bars represent the statistical errors.

$3 \%$ systematic uncertainty in the prediction of the Far to Near $\hat{I}_{i}$ e ratio. In anti-neutrino focusing, twice as much exposure $\left(0.9 \cdot 10^{20}\right.$ pot $)$ allows to cover both the LSND region and the new MiniBooNe results. Both favored MiniBooNe parameter sets, corresponding to two different energy regions in the MiniBooNe anti-neutrino analysis, fall well within the reach of this proposal. The sensitivity for $v_{e}$ disappearance in the $\sin ^{2}\left(2 \theta_{\text {new }}\right), \Delta m^{2}$ plane is shown for one data taking year. The oscillation parameter region related to the anomalies from the combination of the published reactor neutrino experiments, GALLEX and SAGE calibration sources experiments is completely 


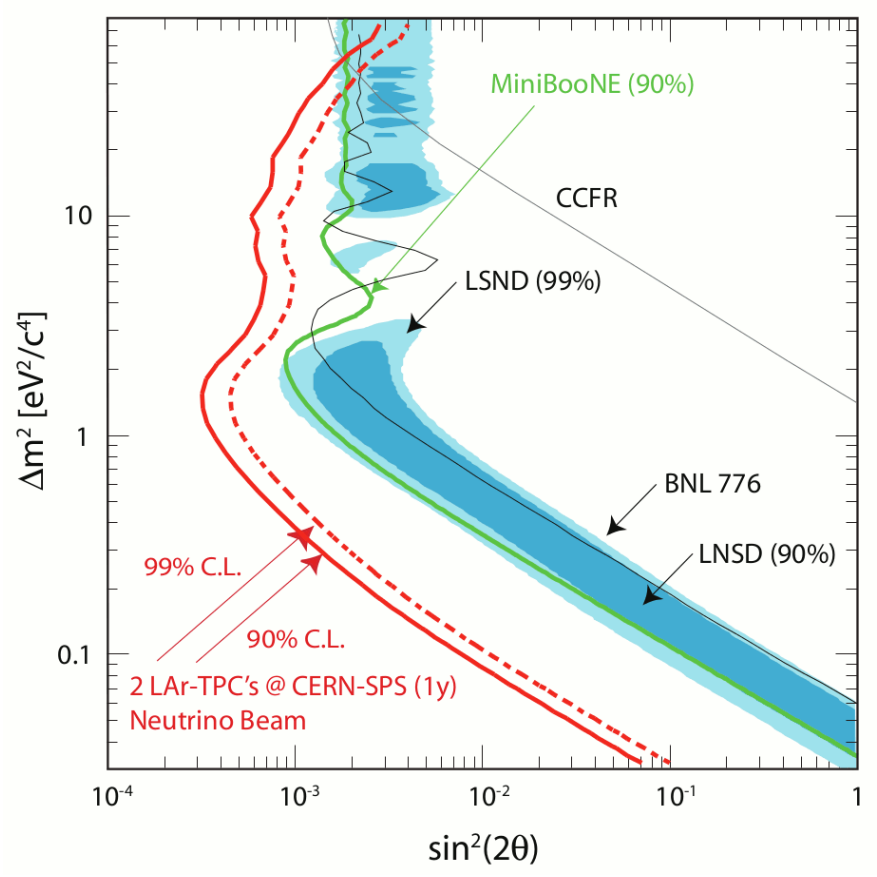

Figure 6: $v_{\mu} \rightarrow v_{e}$ expected sensitivity for the proposed experiment exposed at the CERN-SPS neutrino beam for $4.5 \cdot 10^{19}$ pot (1 year). The LSND allowed region is fully explored.

explored.

The $v_{\mu}$ disappearance signal is well studied by the spectrometers, with very large statistics and full disentangling of $v_{\mu}$ and anti- $v_{\mu}$ interplay. The expected sensitivity for $\sin ^{2}\left(2 \theta_{\text {new }}\right)$ is one order of magnitude better than present SBL experiments at $\left|\Delta m^{2}\right|>2 e V^{2}$, see Figure 7 .

\section{References}

[1] A. Aguilar et al. Evidence for neutrino oscillations from the observation of $\bar{v}_{e}$ appearance in a $\bar{v}_{\mu}$ beam. Phys. Rev. D, 64:112007, Nov 2001.

[2] A. A. Aguilar-Arevalo et al. . Unexplained Excess of Electron-like Events from a 1-GeV Neutrino Beam. Phys. Rev. Lett., 102:101802, Mar 2009.

[3] J. N. Abdurashitov et al. Measurement of the solar neutrino capture rate with gallium metal. III. Results for the 2002-2007 data-taking period. Phys. Rev. C, 80:015807, Jul 2009.

[4] G. Mention et al. Reactor antineutrino anomaly. Phys. Rev. D, 83:073006, Apr 2011.

[5] C. Giunti and M. Laveder. Short-Baseline Electron Neutrino Disappearance, Tritium Beta Decay and Neutrinoless Double-Beta Decay. Phys.Rev., D82:053005, 2010.

[6] M. Antonello et al. Search for anomalies from neutrino and anti-neutrino oscillations at $\Delta m^{2} \sim 1 e V^{2}$ with muon spectrometers and large LAr-TPC imaging detectors. arXiv:1203.3432v2. 


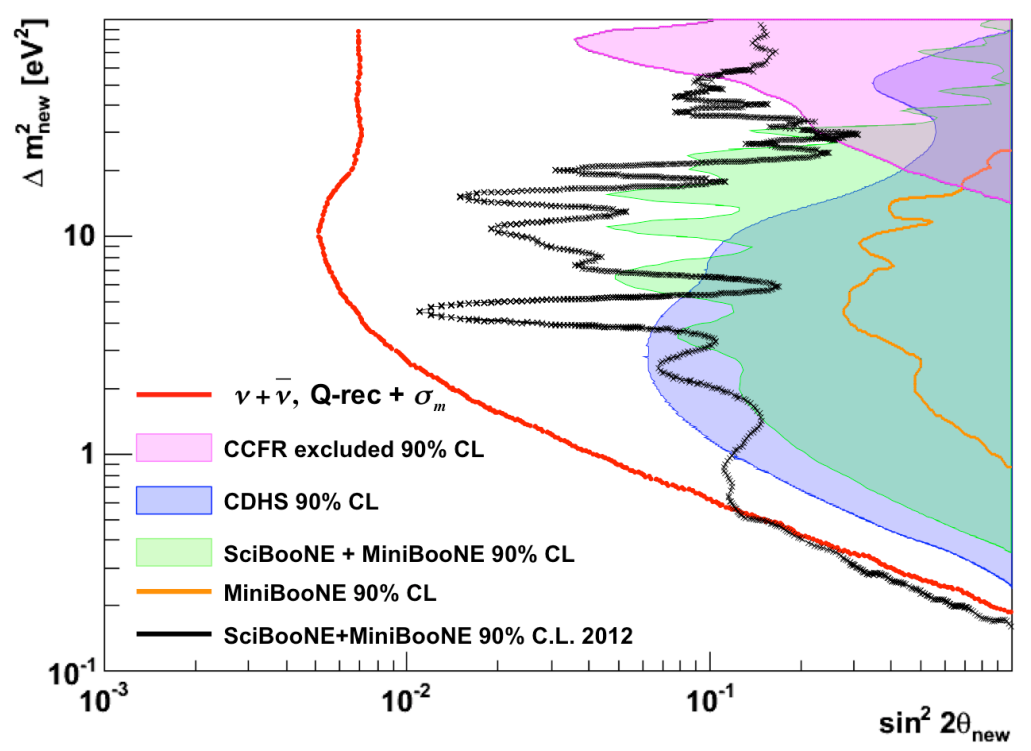

Figure 7: $v_{\mu}$ disappearance sensitivity. The red line represents the sensitivity plot at $90 \%$ C.L. for the $v_{\mu}$ disappearance considering three years of data taking in the CERN-SPS beam, 2 years in anti-neutrino and 1 year in neutrino mode. The limit is obtained from CC events fully reconstructed in NESSiE+LAr. 